\title{
ANÁLISE COMPARATIVA DO PROCESSO DE IDENTIFICAÇ̃̃O AUTOMATIZADA DE MEMBROS FINAIS A PARTIR DE IMAGENS COM DIFERENTES RESOLUÇÕES ESPECTRAIS PARA A REGIÃO DE NIQUELÂNDIA (AVIRIS, ETM+ E ASTER)
}

\author{
Osmar Abílio de Carvalho Júnior ${ }^{1}$, Renato Fontes Guimarães², Éder de Souza Martins³, \\ Ana Paula Ferreira de Carvalho ${ }^{4}$ e Roberto Arnaldo Trancoso Gomes ${ }^{5}$ \\ Recebido em 21 fevereiro, 2005 / Aceito em 17 junho, 2005 \\ Received on February 21, 2005 / Accepted on June 17, 2005
}

\begin{abstract}
The spectral mixture concept allowed a new approach on image classification procedures. This approach comprehends three processing stages: (a) endmembers detection, (b) elements location and (c) quantification. The aim of this work was to develop a comparative analysis of endmembers identification from an automatic process using images with different spectral resolutions: AVIRIS, Landsat ETM+ and ASTER. Endmembers detection techniques were developed to hyperspectral images but are being used to multispectral sensors. Thus it is important to evaluate limitations for the use of lower spectral resolution images. The selected area is a natural Savanna region (locally named Cerrado) in Niquelândia (GO), Brazil. It was used a hyperespectral image from the AVIRIS sensor that was spectrally resampled to Landsat/ETM+ and ASTER sensors. The AVIRIS sensor developed by NASA (National Aeronautics and Space Administration) was the first imaging sensor to measure the solar reflected spectrum from $400 \mathrm{~nm}$ to $2500 \mathrm{~nm}$ at $10 \mathrm{~nm}$ intervals. Endmembers' automatic identification from the ENVI software comprehends the following steps: a) spectral reduction by the Minimum Noise Fraction (MNF) transformation, b) spatial reduction by the Pixel Purity Index (PPI) and c) manual identification of the members using the N-dimensional visualizer. The comparison among the three sensors was done using PPI images. In spite of PPI image differences it can be seen similarities among existing targets. That similarity is due to vegetation targets predominance on these images. These endmembers were relative to photosynthetic and non-photosynthetic vegetations.
\end{abstract}

Keywords: endmember, pixel purity index, image processing, remote sensing, hyperspectral.

RESUMO. A análise de mistura espectral permite novos avanços nos procedimentos de classificação de imagens. Esta análise compreende três estágios de processamento: (a) Detecção dos membros finais, (b) Localização dos elementos e (c) Quantificação. 0 objetivo deste artigo é desenvolver uma análise comparativa de identificação dos membros finais a partir de um procedimento automatizado usando imagens com diferentes resoluções espectrais: AVIRIS, Landsat ETM+ e ASTER. As técnicas de detecção de membros finais foram desenvolvidas para imagens hiperespectrais, porém, foram usadas para sensores multispectral. Desta forma, é importante avaliar as limitações para o uso de sensores de mais baixa resolução espectral. A área selecionada foi uma região de Cerrado em Niquelândia (G0), Brasil. Foi utilizada uma imagem hiperespectral do sensor AVIRIS que foi espectralmente reamostrado para os sensores Landsat/ETM+ e ASTER. 0 sensor AVIRIS desenvolvido pela NASA (National Aeronautics and Space Administration) foi o primeiro sistema imageador capaz de medir o espectro refletido solar de $400 \mathrm{~nm}$ até $2500 \mathrm{~nm}$ em intervalos de $10 \mathrm{~nm}$. A identificação automática dos membros finais, feita a partir do programa ENVI, compreende as seguintes etapas: a) Redução espectral pela transformação Minimum Noise Fraction(MNF), b) Redução espacial a partir do Pixel Purity Index (PPI) e c) Identificação manual dos membros utilizando o N-dimensional visualizer. A comparação entre os três sensores foi feita usando as imagens PPI. Observa-se que apesar das diferenças espectrais existe uma forte correlação entre os alvos existentes. Essa característica se deve a maior parte da imagem conter basicamente diferenças espectrais de vegetação.

Palavras-chave: membros finais, índice de pureza do pixel, processamento de imagem, sensoriamento remoto, hiperespectral.

1,2 Universidade de Brasília, Departamento de Geografia, Campus Universitário Darcy Ribeiro, Asa Norte, 70910-900 Brasília, DF, Brasil. Fone: 3071859; Fax: (61) 2721909 - E-mails: osmarjґunb.br; renatofg@unb.br

3 EMBRAPA Cerrados, Rodovia Brasília Fortaleza, km 18 Planaltina Distrito Federal. E-mail: eder@cpac.embrapa.br

4 Universidade de Brasília, Departamento de Ecologia, Campus Universitário Darcy Ribeiro - ICC Centro, 70910-900 Brasília, DF, Brasil. Fone: (61) 367-5001; Fax: (61) 2721909 - E-mail: anapaula@unb.br

5 Departamento de Geografia, Universidade Federal do Rio de Janeiro (UFRJ) - E-mail: ratgomes@ig.com.br 


\section{INTRODUÇ̃̃o}

0 conceito de mistura espectral traz uma nova abordagem ao processamento digital de imagens. Esse tipo de análise busca identificar e quantificar os elementos que compõem um determinado pixel. Três questões principais são abordadas na análise de mistura espectral de imagens: (a) Quais os elementos e os respectivos espectros que compõem a imagem? (b) Onde está o elemento procurado? e (c) Quanto do elemento existe em cada pixel? (Carvalho Júnior et al., 2003). A partir desses questionamentos pode-se definir um encadeamento metodológico para 0 processamento de imagens com o propósito de descrever a mistura espectral formado por três etapas: (a) detecção dos membros finais ou membros puros de forma automatizada, (b) emprego de um classificador espectral para identificação e localização do espectro procurado, e (c) emprego de um índice morfométrico do espectro para estimar sua abundância relativa dentro do pixel.

Os membros finais consistem nos elementos puros na imagem que, por mistura, formam todos os demais espectros presentes. As técnicas para a detecção dos membros finais foram desenvolvidas para os sensores hiperespectrais, mas vêm sendo empregada para sensores multiespectrais. Portanto, é importante avaliar as limitações para o emprego em sensores multiespectrais, que apresentam resolução espectral mais baixa.

0 presente trabalho possui como objetivo avaliar e comparar a detecção dos membros finais de forma automatizada para diferentes sensores em ambiente de cerrado. Para tanto, será utilizada imagem hiperespectral do sensor Airborne Visible/InfraRed Imaging Spectrometer (AVIRIS) e, a partir dela, serão simuladas imagens referentes ao sensor ASTER e LANDSAT/ETM+

\section{Representação Geométrica das Misturas Espectrais}

A disposição dos pixels de uma imagem em um espaço ndimensional de atributos, no qual seus eixos apresentam-se sem correlação e em sua inerente dimensionalidade (como no caso das componentes da Análise de Principais Componentes - APC e Minimum Noise Fraction - MNF), gera figuras geométricas de conjuntos convexos que descrevem os membros finais e os produtos provenientes de sua mistura (Smith et al., 1985; Bateson \& Curtiss, 1993 e 1996; Boardman, 1993).

Normalmente, verifica-se uma correlação da figura geométrica, gerada pela nuvem dos pixels, com 0 número de eixos presentes. Denota-se que, em um espaço $\mathrm{n}$ - dimensional, a nuvem de pixels forma uma figura que possui $(n+1)$ vértices, $(n+1)$ lados e $(n-1)$ dimensões. Desta forma, quando os dados ficam adequados em uma distribuição em 2-d é gerado um triângulo com 3 vértices e 3 lados, enquanto que para um simplex 3-d é gerado um tetraedro com 4 vértices e 4 lados e, assim, sucessivamente (Figura 1).

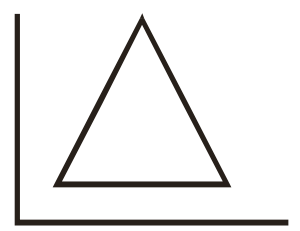

$$
\text { Espaço Bidimensional }
$$

Simplex 2D: um triângulo

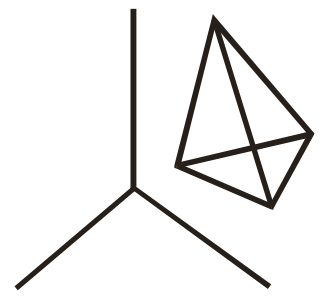

Espaço Tridimensional

Simplex 3D: um tetraedro

Figura 1 - Demonstração do conjunto convexo gerado de acordo com a dimensão do espaço.

Todos os pontos do interior do conjunto convexo podem ser considerados como combinações dos seus vértices. Desta forma, como retratação do espaço espectral, a figura geométrica exibe nos seus vértices os membros finais ou puros, enquanto os demais pixels são provenientes de suas misturas. Isto representa uma notável simplificação porque, ao invés de procurarmos a solução ótima para todos os pixels da imagem com várias soluções viáveis, podemos restringir a procura a alguns pontos especiais, os vértices.

Essa constatação permite descrever o processo de mistura dentro de uma óptica geométrica onde a posição relativa de um determinado pixel em relação aos vértices determina a porcentagem existente de cada membro final. Esse procedimento consiste na base para os métodos de análise linear de mistura proposta por Smith \& Adams (1985 a, b).

\section{Membros Finais Verdadeiros, da Imagem e Virtuais}

A diferença conceitual entre os membros finais da imagem, verdadeiros e virtuais é importante para se compreender as limitações e os ajustes necessários a serem empregados na geometria da análise de mistura.

Os membros finais verdadeiros correspondem aos espectros puros dos materiais que compõem a cena, sendo obtidos em laboratório ou em campo com a medição espectrorradiométrica individual do material.

Os membros finais da imagem são os espectros mais puros presentes no conjunto dos pixels analisados. Como os pixels correspondem a um sinal de uma área, muitas vezes, inexiste na 
imagem um pixel puro com a presença total do material procurado. Nesse caso, obtém-se um espectro de uma mistura, onde existe uma maior proporção do material de interesse, que difere do membro final obtido em laboratório que realmente representa o material puro. A Figura 2 ilustra o posicionamento desses tipos de membros finais e seus respectivos arranjos, uma referente aos membros finais da imagem (linha tracejada) e outra obtida pelos membros finais verdadeiros (linha contínua). Verifica-se que 0 simplex da imagem está incluso dentro do simplex verdadeiro. Os vértices obtidos pela imagem A', B' e C' são relativamente os pontos com maior contribuição dos respectivos componentes reais $\mathrm{A}$, B e C. Como conseqüência, os vértices da imagem posicionamse de forma a obter a menor distância entre a nuvem dos dados e os vértices reais. Assim os vértices obtidos com a imagem podem ser descritos como mistura dos membros finais verdadeiros podendo-se, assim, retratar o ponto B' como uma mistura de aproximadamente $80 \%$ do membro B e $20 \%$ dos membros A e C (Figura 2). A igualdade para os dois tipos de vértices ocorre apenas quando existe na imagem um pixel completamente ocupada por um material puro. Assim, no caso de todos os espectros puros estarem representados na imagem, haverá coincidência com os membros finais verdadeiros. Na inexistência dessa condição sempre haverá diferença entre os dois tipos de arranjo espacial.

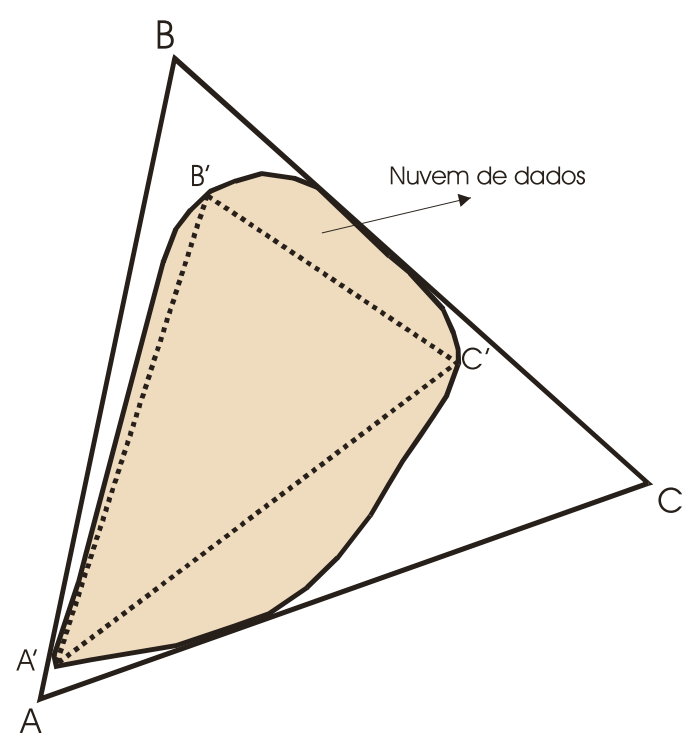

Figura 2 - Demonstração do simplex proveniente dos membros finais da imagem ( $\left.A^{\prime} B^{\prime} C^{\prime}\right)$ e dos membros finais verdadeiros $(A B C)$.

No propósito de obter refinamentos dos membros finais Tompkins et al. (1997) utilizam o modelo de análise linear de mistura espectral e 0 erro médio quadrático como parâmetro para discriminar o grau de ajuste dos membros finais ao arranjo espacial dos dados. A partir disso, os autores formulam um método para encontrar novos membros finais com um posicionamento que reduza 0 erro de ajuste do simplex aos dados, de forma a obter resultados mais fidedignos matematicamente. Os novos membros, que se posicionam fora da nuvem de dispersão dos dados, são denominados de pontos virtuais.

Desta forma, existem as seguintes formas para selecionar os membros finais: (a) determinar os membros finais presentes na imagem, (b) utilizar espectros provenientes de bibliotecas espectrais que representariam espectros verdadeiros e (c) emprego de modelos matemáticos de mistura linear que objetivam detectar membros finais virtuais que apresentem uma maior adequação com a nuvem de dados.

Todos os métodos apresentam vantagens e desvantagens. 0 primeiro método apresenta facilidades para procedimentos de automatização uma vez que os membros finais estão contidos dentro dos dados em análise, no entanto dificilmente representam os membros verdadeiramente puros. No segundo método uma das principais dificuldades é que os espectros da biblioteca podem não se ajustar às variações sazonais de determinados alvos como, por exemplo, a vegetação, podendo existir um descompasso entre o espectro do campo e o espectro da imagem (Garcia \& Ustin, 2001), requerendo um amplo estudo de campo com levantamento dos espectros puros presentes na cena. 0 terceiro método necessita de informações iniciais para determinar os espectros virtuais e 0 ajuste matemático pode divergir da realidade.

No entanto, a definição dos membros finais da imagem é um importante passo para automatização do processo, inclusive para os demais procedimentos. A detecção dos membros finais da imagem permite, por classificação espectral, identificar na biblioteca espectral quais são os espectros puros equivalentes. Além disso, permite identificar os locais mais adequados para levantamentos de campo. No caso da modelagem dos membros finais virtuais, os membros finais da imagem podem servir como informações iniciais ao modelo.

\section{Algoritmos}

Os algoritmos usados para detectar os membros finais implicitamente ou explicitamente assumem a geometria do conjunto convexo aplicado no modelo linear de mistura (Batenson et al., 2000; Batenson \& Curtis, 1996; Berman et al., 2004; Winter, 1999; Tompkins et al., 1997). Provavelmente, o modelo mais utilizado seja 0 algoritmo desenvolvido por Boardman \& Kruse (1994) devido, em parte, ao fato de estar incorporado no pacote de processamento hiperespectral Environment for Visualizing Images - ENVI (ENVI, 2000) amplamente utilizado no processamento 
de imagens hiperespectrais (Carvalho Júnior et al., 1999; Kruse, 1996; 1999; Kruse \& Boardman, 1999; Kruse et al., 1996; Richardson, 1996; Richardson et al., 1994) como de imagens multiespectrais com bons resultados (Carvalho et al., 2002; Bloise et al., 2003).

A metodologia de Boardman \& Kruse (1994) para a detecção dos membros finais apresenta três etapas: (a) redução da dimensão espectral (Minimum Noise Fraction - MNF), (b) redução da dimensão espacial (Purity Pixel Index - PPI), e (c) identificação manual (utilizando um visualizador n-dimensional). Essa metodologia visa eliminar os fatores redundantes, tanto espaciais como espectrais, que nada alteram o conjunto de soluções viáveis para os membros finais.

\section{Área de Estudo}

A área de estudo localiza-se no estado de Goiás, município de Niquelândia, no Maciço Máfico-Ultramáfico de Niquelândia, que contém importantes reservas de minério de níquel. 0 acesso se dá, a partir de Brasília, pela rodovia Belém - Brasília (BR-153) até a cidade de Uruaçu. Entre Uruaçu e Niquelândia o percurso é de 90 km em estradas asfaltadas (Figura 3).

A distribuição e fisionomia da vegetação no Maciço de Niquelândia apresentam um forte controle geológico, descrito por Brooks et al. (1990). Nessa região, extensas áreas de rochas ultramáficas são cobertas por vegetação herbácea dominada por gramíneas enquanto as rochas gabróicas apresentam vegetação arbórea. 0 fogo que ocorre durante os meses mais secos (junho agosto) influencia em certo grau a fitofisionomia da vegetação. № entanto, isoladamente, o fogo não explica o limite abrupto entre as rochas gabróicas (máficas) e o substrato ultramáfico (Carvalho et al., 2001).

Diferentes graus de floresta aberta ou floresta baixa (mata) podem ser encontrados ao longo dos cursos d'água e vales. A existência dessas áreas demonstra a habilidade de muitas espécies tolerarem os solos provenientes de rochas ultramáficas, desde que se tenha profundidade e umidade nos solos adequados, além da proteção ao fogo.

Nesse tipo de ambiente foi descoberta a primeira hiperacumulação de níquel da América do Sul. Uma espécie de Cnidosculus, descrita por C. bahianus, com escrube de 1,5 a $2 \mathrm{~m}$, de folhas e frutos espinhosos, produz grandes quantidades de látex branco, na qual foi posteriormente encontrado 1,35\% de níquel na matéria seca. Esse comportamento é similar ao da Sebertia acuminata (Sapotaceae) que ocorre na região de serpentinitos da Nova Caledônia, que possui látex azul esverdeado contendo mais de 25\% de Ni na matéria seca e 1,17\% nas folhas. As folhas de Cnidosculus também são menos ricas em látex, com apenas 0,01-0,11\%. Essas espécies são similares a $C$. vitjfolius altamente difundida em Americano do Brasil e Barro Alto. Entretanto, estudos em solos com baixa concentração de níquel são necessários para revelar as relações entre essas taxas.

As plantas não ultrapassam $1 \mathrm{~m}$ de altura nas áreas mais altas do maciço utramáfico. Entre as plantas mais proeminentes estão as espécies de Paepalanthus (Eriocaulaceae), Heteropteris (Malpighiaceae) e Vellozia (Velloziaceae). Diversas espécies de Vellozia (canela-de-ema) ocupam variados substratos nas maiores altitudes no Brasil central.

0 AVIRIS foi trazido para o Brasil em 1995 na missão SCAR-B (Smoke, Clouds and Radiation - Brazil), que teve como propósito avaliar efeitos atmosféricos. Essa atividade foi uma operação conjunta da NASA, INPE (Instituto Nacional de Pesquisas Espaciais) e a AEB (Agência Espacial Brasileira) (Kaufman et al., 1998). Durante a missão SCAR-B, o sensor AVIRIS sobrevoou a região de Niquelândia, no dia 16 de agosto de 1995, imageando áreas com concentração supergênica de minério, no caso, de níquel laterítico com efeitos sobre a vegetação. A linha de vôo foi realizada longitudinalmente ao complexo de Niquelândia, cruzando as unidades geológicas. A imagem em estudo apresenta uma dimensão de 240 colunas por 356 linhas contendo 85440 pixels contendo tanto áreas vegetadas como solo desnudo (Figura 4).

\section{METODOLOGIA}

\section{Correção Atmosférica}

A utilização de imagens de sensores de alta resolução espectral requer processamentos que reduzam ou compensem os efeitos atmosféricos, uma vez que diferentes fenômenos de absorção e espalhamento de gases por moléculas e aerossóis afetam o sinal recebido pelo sensor (Tanré et al., 1990).

Os procedimentos desenvolvidos para correção atmosférica de imagens multiespectrais caracterizam-se por serem modelos do tipo ordinário discreto onde se obtém um único valor de correção para toda a cena em estudo. Com o advento da espectroscopia de imageamento, verifica-se uma tendência para modelos de correção atmosférica do tipo distribuído, onde é realizado um tratamento específico de pixel para pixel. Este avanço foi possível devido à capacidade de detecção das feições de absorção dos elementos atmosféricos pelos sensores hiperespectrais que fornecem uma estimativa da quantidade de gases e aerossóis presentes (Green, 1990, 1991; Carrere \& Conel, 1993; Gao et al., 1993; Schmid, 1996). Estes modelos permitem visualizar a varia- 


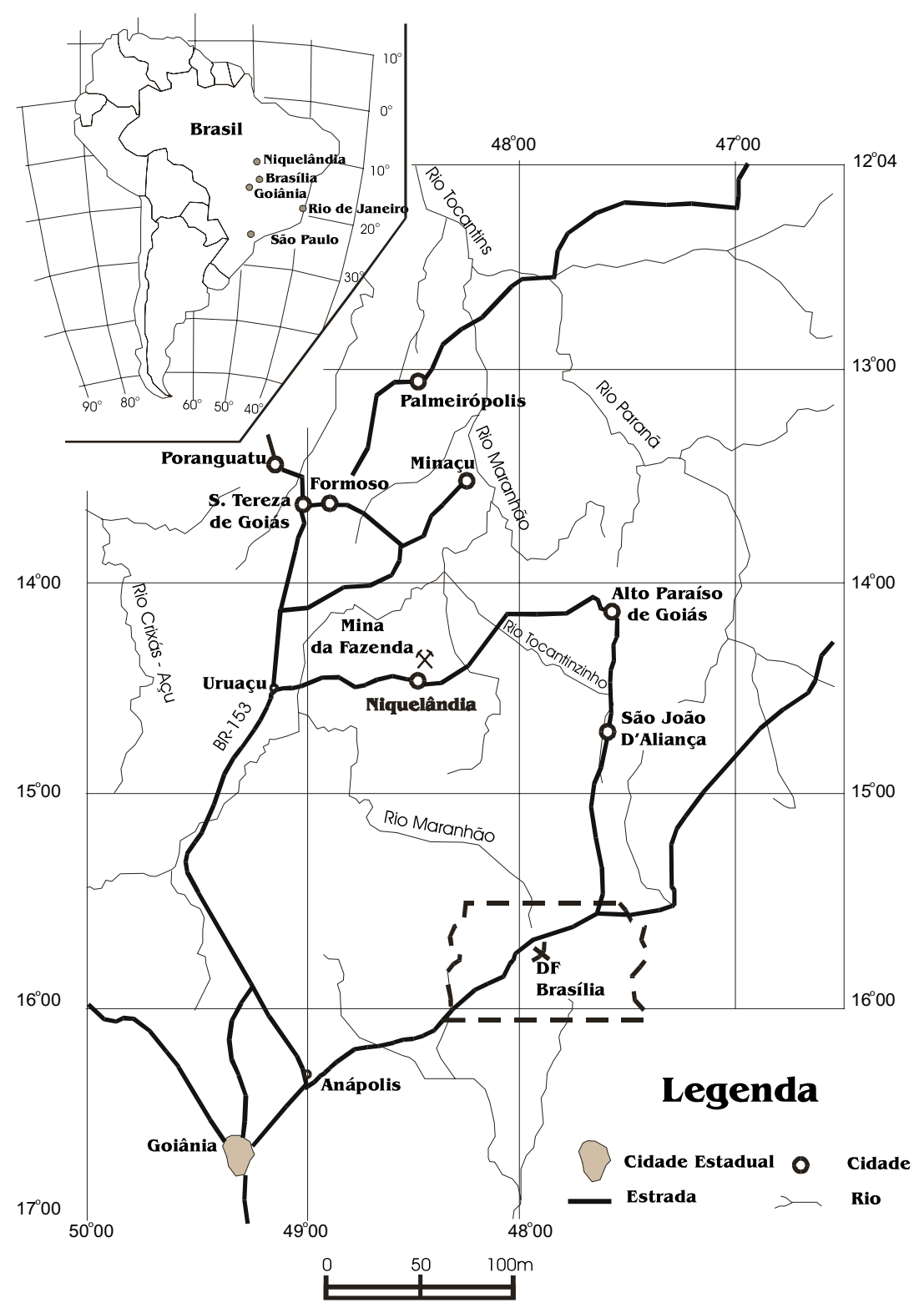

Figura 3 - Localização da área de estudo.

bilidade dos parâmetros atmosféricos ao longo do tempo, espaço e altitude.

Portanto, é fundamental na formulação dos modelos o conhecimento das propriedades ópticas da atmosfera bem como do processo de interação da radiação na trajetória entre o sensor e a superfície (Latorre et al., 2002). Estes métodos são fundamentados na teoria da transferência radiativa (Chandrasekhar, 1960), sendo os mais difundidos os códigos: Simulation of Satellite Signal in the Solar Spectrum-5S (Tanré et al., 1986 e 1990), Second Simulation of Satellite Signal in the Solar Spectrum $6 \mathrm{~S}$ (Vermote et al., 1996), Low Resolution Atmospheric Radiance and Transmittance Model- LOWTRAN (Isaacs \& Vogelmann, 1988) e Moderate Resolution Atmospheric Radiance and Transmittance Model - MODTRAN (Adler-Golden et al., 1998; Anderson et al., 1995; Berk et al., 1996, 1989).

No presente trabalho é utilizado o método de correção atmosférica proposto por Green (1990), formulado especificamente para as imagens hiperespectrais AVIRIS. Esse método proporciona uma estimativa dos parâmetros atmosféricos e cálculo da reflectância aparente da superfície usando o código de transferência 
radiativa MODTRAN 3 (Berk et al., 1989; Anderson et al., 1995) em conjunto com um modelo de ajuste não linear por mínimos quadrados (Green et al., 1991, 1993).

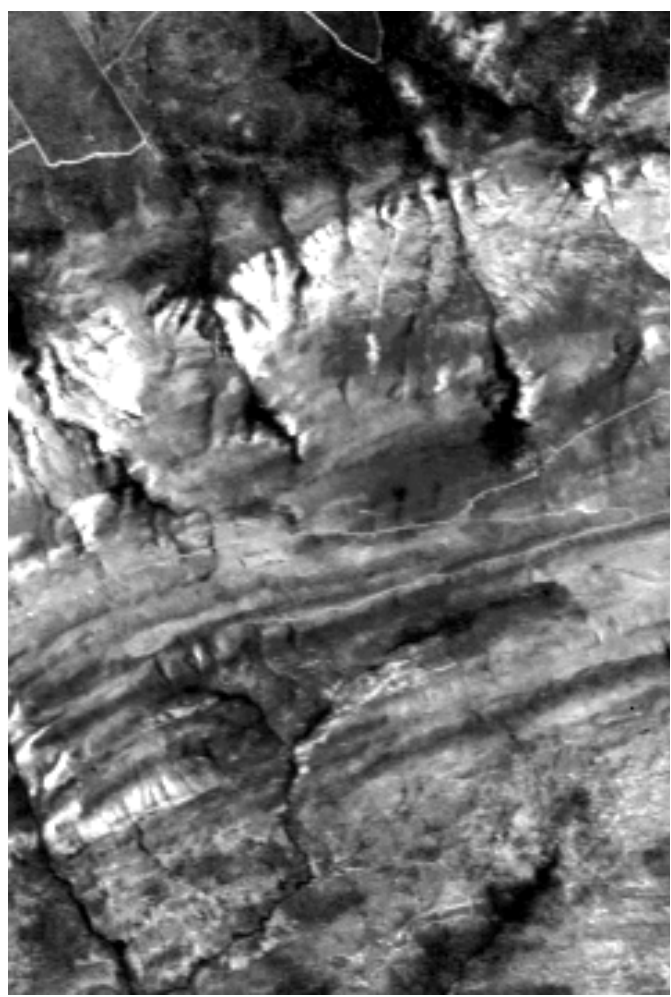

Figura 4 - Imagem do sensor AVIRIS da área de estudo, banda 30 corresponde ao comprimento de onda de $0.6579 \mu \mathrm{m}$.

\section{Reamostragem dos Espectros do Sensor AVIRIS para LANDSAT/ETM + e ASTER}

Os espectros da imagem AVIRIS foram reamostrados para as bandas dos sensores LANDSAT/ETM+ e ASTER com o propósito de realizar a comparação entre os sensores. Desta forma, as características ambientais e os processos de correção atmosférica permanecem os mesmos não alterando a análise sobre os membros finais.

Para tanto, foram utilizados filtros específicos que descrevem os comportamentos dos sensores em função do comprimento de onda (Figura 5). Este procedimento permite gerar as imagens ASTER e Landsat $7 / E T M+$ a partir de uma mesma imagem AVIRIS.

Para facilitar a visualização a Figura 6 apresenta os espectros obtidos para os três sensores para a vegetação fotossinteticamente ativa (VFA) e vegetação não fotossinteticamente ativa (VNFA).

\section{Identificação dos Membros Finais}

Na metodologia de Boardman \& Kruse (1994) a diminuição da dimensionalidade das imagens é obtida pelo método MNF (Green et al., 1988). Esse método, além de possibilitar a eliminação do ruído, permite a diminuição da dimensionalidade da imagem em eixos descritos pelos materiais ou feições físicas ambientais que constituem a cena. No entanto, deve-se atentar que os novos eixos não estão necessariamente sempre correlacionados com um material específico ou uma variável física. É sempre requerida uma interpretação cautelosa na ligação entre um determinado eixo com uma componente da imagem (Carvalho Júnior et al., 2003). 0 emprego de técnicas de redução de dimensionalidade em imagens hiperespectrais proporciona uma intensa diminuição das bandas iniciais introduzidas devido à alta redundância de informação.

No presente trabalho foram definidas as bandas referentes às informações para serem submetidas ao PPI pela relação visual sinal/ruído. Para as imagens ASTER e LANDSAT 7/ETM+ foram consideradas as cinco primeiras componentes MNFs. Em relação às imagens AVIRIS, onde ocorre um aumento gradual do ruído, considerou-se duas situações: (a) as onze primeiras componentes e (b) as seis primeiras componentes.

A identificação do membro final não é um processo simples, pois raramente os pixels $100 \%$ puros estão presentes no conjunto de dados da imagem, implicando na formação de um simplex com vértices menos pronunciados e mais difíceis de serem detectados. Desta forma, na maioria das vezes, a distribuição dos dados não apresenta uma forma geométrica tão definida e a simples análise de um gráfico de dispersão pode não representar todas as combinações possíveis, restando ainda muitas possibilidades para a reconstrução do simplex (Carvalho Júnior et al., 2002).

Um procedimento que permite um detalhamento dos vértices do simplex pode ser facilmente visualizado em um espaço bidimensional (Craig, 1994). Primeiramente, determina-se para os dados um primeiro invólucro com $n+1$ vértices que demarcam um simplex inicial (Figura 7). Deve-se atentar que esses pontos não representam, necessariamente, os pontos mais extremos. Como segundo passo, inscreve-se um novo simplex de segunda ordem com $n+1$ vértices na posição oposta aos dos vértices do simplex de $1^{\text {a }}$ ordem, ou seja, no ponto absoluto mais proeminente da face oposta do vértice do $1^{\circ}$ simplex (Figura 7 ).

Em decorrência, o emprego dessa técnica em um espaço ndimensional pode gerar inúmeros vértices. Uma maneira de selecionar os mais relevantes é o emprego de um índice. Dentro dessa concepção, o Índice de Pureza do Pixel (PPI) busca esti- 

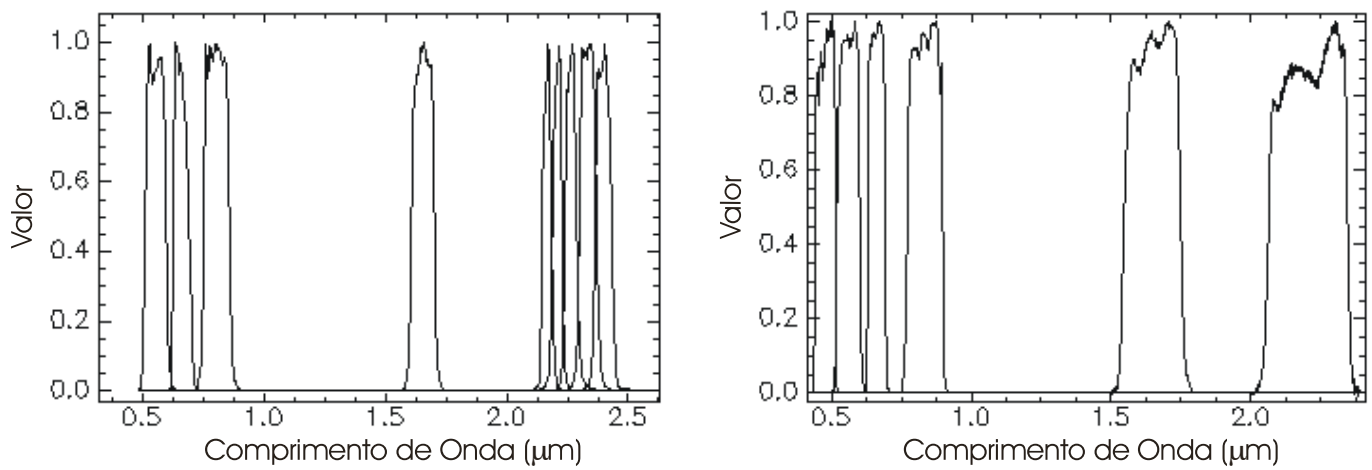

Figura 5 - Filtros dos sensores ASTER e LANDSAT7/ETM+.

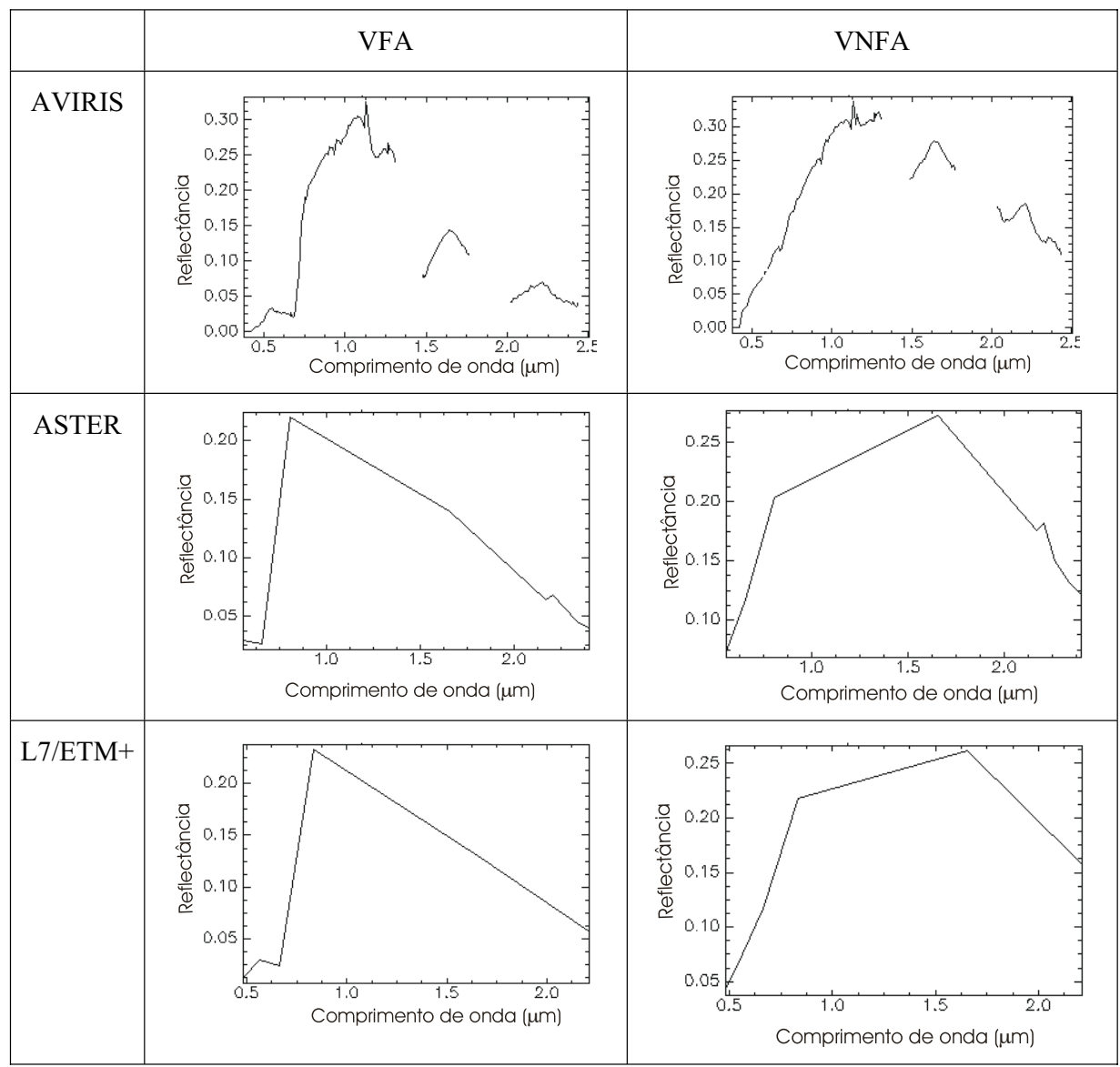

Figura 6 - Comparação entre os espectros dos sensores AVIRIS, ASTER e LANDSAT7/ETM+ para a vegetação fotossinteticamente ativa (VFA) e vegetação não fotossinteticamente ativa (VNFA).

pular o quanto o pixel é extremo dentro do simplex (Boardman \& Kruse, 1994; Boardman et al., 1995).

0 método PPI registra e contabiliza os pixels que são vértices nas diferentes projeções do simplex. Desta forma, os pontos rela- tivamente mais puros tendem a se repetir nas diferentes projeções aumentando 0 seu valor PPI. 0 número de iterações é definido pelo usuário, assim como o valor mínimo de PPI para que o pixel seja considerado como puro. Quanto maior for o número 

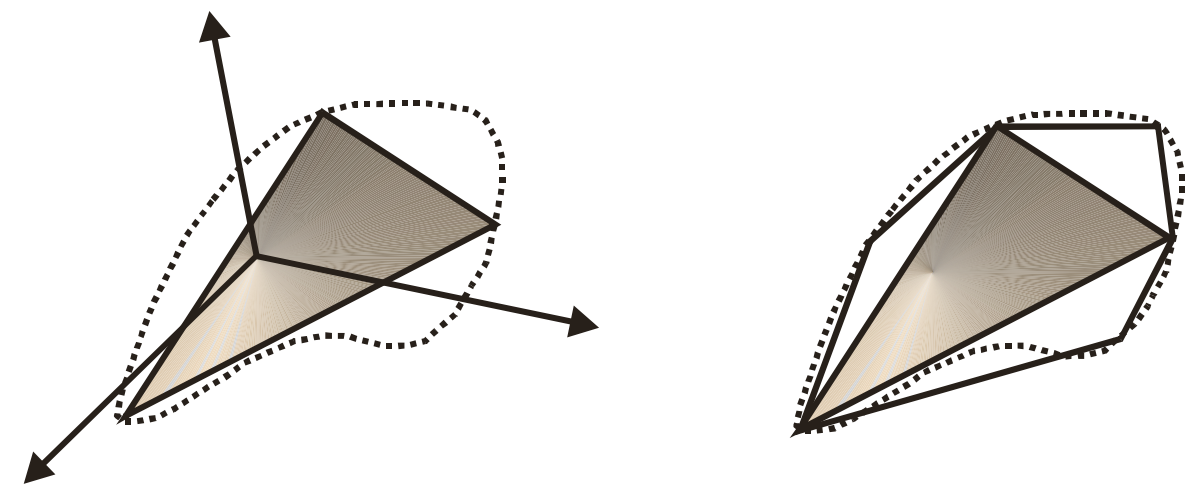

Figura 7 - Definição dos pontos referentes aos vértices do Simplex: (a) vértices de $1^{\mathrm{a}}$ ordem, (b) vértices de $2^{\mathrm{a}}$ ordem. (Fonte: Craig, 1994)

de iterações estipuladas, mais fidedigna é a informação obtida. Para averiguar se o número de iterações foi apropriado utiliza-se um gráfico que contrapõe 0 valor do número de iterações com 0 número de pixels que obedecem à condição de PPI estipulado. Quando a curva do gráfico torna-se próxima de um patamar significa que o número de iterações é satisfatório para a análise.

0 procedimento PPI também gera uma imagem que apresenta, para cada pixel, o valor do número de vezes em que esse foi definido como vértice. Quanto maior for o valor do pixel na imagem PPI maior é o seu grau de pureza. Desta forma, a partir da imagem PPI pode-se estipular novos valores para corte utilizando a técnica de fatiamento, o que permite uma melhor seleção dos membros finais da imagem. Observa-se que, na medida em que aumenta o valor de PPI de corte, as áreas com pixels puros tornam-se mais restritas. Esse procedimento é bastante útil na averiguação de campo, pois permite determinar a localização dos pixels puros.

Para o presente trabalho foi definido como valor de corte os cem maiores valores de PPI para cada sensor analisado. No caso da imagem AVIRIS, devido à maior presença de componentes MNF com sinal foram consideradas duas situações para o cálculo do PPI: (a) onze primeiras componentes e (b) seis primeiras componentes. Desta forma, pode-se avaliar a influência das componentes com aumento relativo de ruído.

\section{RESULTADOS DA COMPARAÇ̃̃ ENTRE OS SENSORES}

A Tabela 1 apresenta 0 número de pixels coincidentes obtidos pelo procedimento PPI para os diferentes sensores. A comparação referente aos cem maiores valores de PPI apresentou uma intersecção de praticamente a metade dos pixel entre o sensor hiperespectral em relação aos multiespectrais. Entre as imagens multiespectrais do sensor ASTER e Landsat 7/ETM+ observa-se uma alta coincidência dos membros finais.

Considerando os pontos obtidos pelo PPI para o sensor ASTER e AVIRIS observa-se que os membros finais selecionados são referentes aos mesmos grupos de espectros. São identificados os seguintes grupos: vegetação fotossinteticamente ativa, vegetação não fotossinteticamente ativa e espectros de solos com a presença da feição de absorção do mineral caulinita. Os membros finais não coincidentes identificados pelo PPI são também pertencentes aos mesmos grupos descritos com pequenas diferenciações provenientes de mistura e aumento da componente sombra o que tornam os membros finais encontrados equivalentes. Os membros finais identificados no LANDSAT/ETM+ apresentam também todos os grupos espectrais compatíveis com os obtidos pelo sensor AVIRIS.

A Figura 8 apresenta uma série de espectros relativos aos principais grupos espectrais identificados nos 100 maiores valores de PPI da imagem Landsat; ASTER e AVIRIS. Apesar do método PPI identificar diferenciações entre os grupos com maior presença de solo em relação aos de VNFA observa-se para as imagens multiespectrais a dificuldade de distingui-los devido à inexistência das feições de absorção. No presente caso, a principal diferença nos sensores ASTER e LANDSAT/ETM+ para esses espectros de solo é a maior subida da banda 4 para a banda 5 .

De forma complementar, foram analisados separadamente os pixels do sensor AVIRIS que não foram detectados pelo sensor ASTER e LANDSAT/ETM+. Nesses não foram identificados nenhum membro final que não tivesse equivalente com os membros finais presentes nos sensores multiespectrais.

\section{CONCLUSÃO}

Observa-se que apesar das diferenças espectrais dos sensores existe uma forte correlação dos membros finais identificados entre 
Tabela 1 - Número de coincidência entre os 100 maiores PPI dos diferentes sensores.

\begin{tabular}{|l|c|c|c|c|}
\hline & $\begin{array}{c}\text { AVIRIS } \\
6 \text { MNFs }\end{array}$ & $\begin{array}{c}\text { AVIRIS } \\
11 \text { MNFs }\end{array}$ & $\begin{array}{c}\text { ASTER } \\
5 \text { MNF }\end{array}$ & $\begin{array}{c}\text { LANDSAT7/ETM+ } \\
5 \text { MNF }\end{array}$ \\
\hline $\begin{array}{l}\text { AVIRIS } \\
6 \text { bandas }\end{array}$ & 100 & 70 & 53 & 53 \\
\hline $\begin{array}{l}\text { AVIRIS } \\
11 \text { bandas }\end{array}$ & 70 & 100 & 50 & 48 \\
\hline $\begin{array}{l}\text { ASTER } \\
5 \text { bandas }\end{array}$ & 53 & 50 & 100 & 72 \\
\hline $\begin{array}{l}\text { LANDSAT7/ETM+ } \\
5 \text { bandas }\end{array}$ & 53 & 48 & 72 & 100 \\
\hline
\end{tabular}
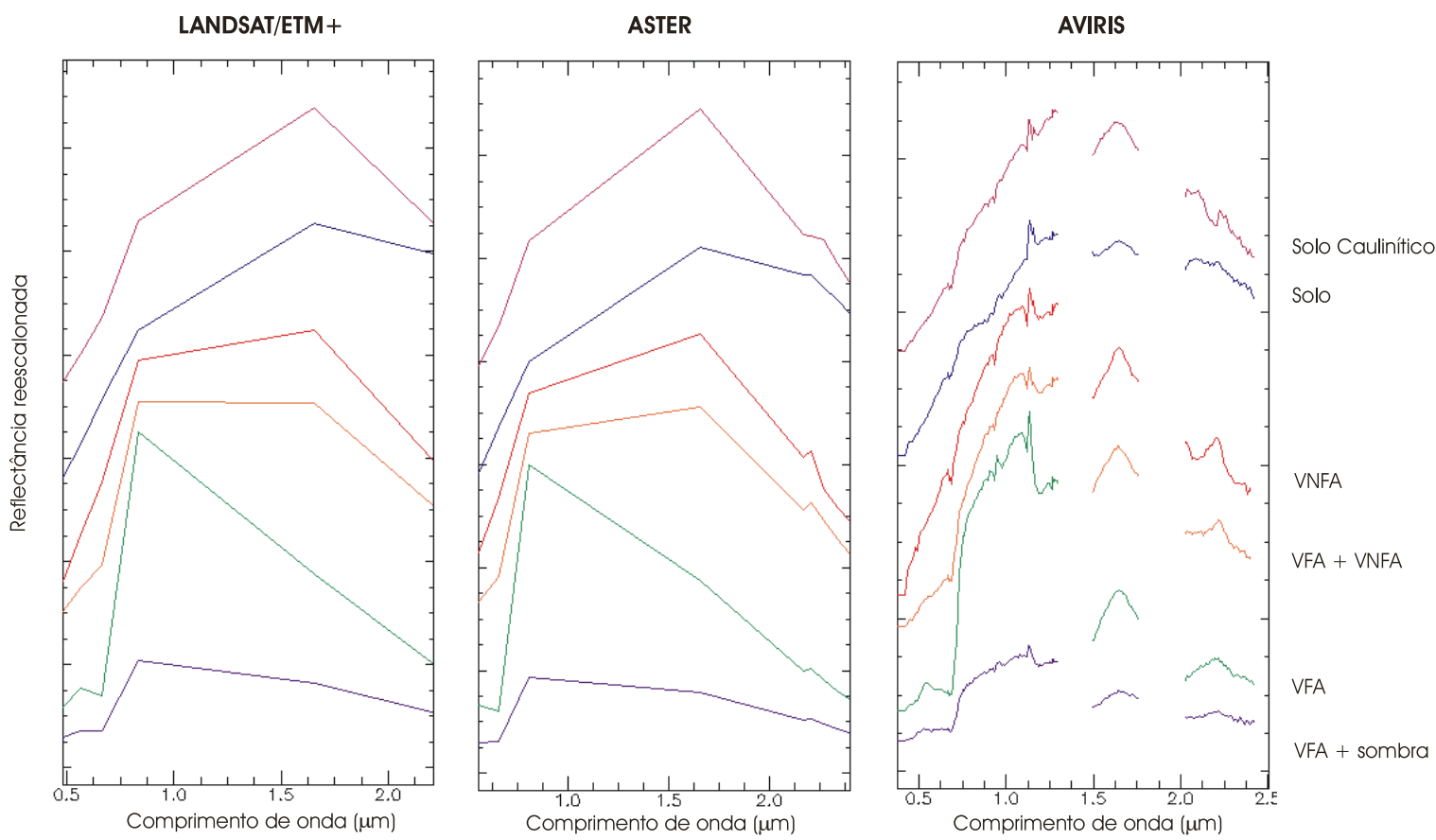

Figura 8 - Espectros relativos aos membros finais obtidos pelo PPI para as imagens ASTER, Landsat/ETM+ e AVIRIS.

os alvos analisados. Essa característica se deve a maior parte da imagem conter basicamente diferenças espectrais de vegetação. Mesmo na imagem Landsat/ETM+ com a menor resolução espectral foi possivel distinguir nos maiores 100 pontos do PPI as principais curvas espectrais.

\section{AGRADECIMENTOS}

Os autores agradecem ao Instituto Nacional de Pesquisas Espaciais (INPE) pela concessão das imagens do sensor AVIRIS, ao CNPq pelo financiamento de bolsas de pesquisa e aos consultores da revista de geofísica.

\section{REFERÊNCIAS}

ADLER-GOLDEN S, BERK A, BERNSTEIN LS, RICHTSMEIER S, ACHARYA PK, MATTHEW MW, ANDERSON GP, ALLRED CL, JEONG LS \& CHETWYND JH. 1998. Flaash a MODTRAN4 Atmospheric correction package for hyperspectral data retrievals and simulations. In: Annual JPL Airborne Earth Science Workshop, 7, Pasadena, CA. Summaries, JPL Publication 97-21: p. 9-14.

ANDERSON GP, WANG J \& CHETWYND JH. 1995. MODTRAN3: An update and recent validations against airborne high resolution interferometer measurements. In: Annual JPL Airborne Earth Science Workshop, 5, Pasadena, CA. Summaries, JPL Publication 95(1): 5-8. 
BATESON CA \& CURTISS B. 1993. A tool for manual endmember selection and spectral unmixing. In: Annual JPL Airborne Geosciences Workshop, 4, Pasadena, CA. Summaries, JPL Publication 93-26, p. 3-6

BATESON CA \& CURTISS B. 1996. A method for manual endmember selection and spectral unmixing. Remote Sensing of Environment, 55: 229-243.

BATESON CA, ASNER GP \& WESSMAN CA. 2000. Endmember bundles: a new approach to incorporating endmember variability into spectral mixture analysis. IEEE Transactions on Geoscience and Remote Sensing 38(2): 1083-1094

BERK A, BERNSTEIN LS \& ROBERTSON DC. 1989. MODTRAN: A moderate resolution model for LOWTRAN7. Final report, GL-TR-0122, AFGL, Hanscomb AFB, MA, 42p.

BERK A, BERNSTEIN LS, ROBERTSON DC, ACHARYA PK, ANDERSON GP \& CHETWYND JH. 1996. MODTRAN cloud and multiple scattering upgrade with application. In: Annual JPL Airborne Earth Science Workshop, 6, Pasadena, CA. Summaries, JPL Publication 96(4): 1-7.

BERMAN M, KIIVERI H, RYAN L, ERNST A, DUNNE R \& HUNTINGTON JF. 2004. ICE: A statistical approach to identifying endmembers in hyperspectral images. IEEE Transactions on Geoscience and Remote Sensing 42(10): 1-11.

BLOISE PLC, GUIMARÃES RF, MARTINS ES, CARVALHO APF \& CARVALHO JÚNIOR OA. 2003. Análise de mistura espectral de imagens ASTER no Campo de Instrução Militar de Formosa. In: Simpósio Brasileiro de Sensoriamento Remoto, 11, Belo Horizonte (MG). Anais XI SBSR. São José dos Campos: INPE, p. 2311-2318.

BOARDMAN JW. 1993. Automated spectral unmixing of AVIRIS data using convex geometry concepts: In: Annual JPL Airborne Geosciences Workshop, 4, Pasadena, CA. Summaries, JPL Publication 93-26, p. 11-14.

BOARDMAN JW \& KRUSE FA. 1994. Automated spectral analysis: A geologic example using AVIRIS data, north Grapevine Mountains, Nevada: In: ERIM - Thematic Conference on Geologic Remote Sensing, 10, Environmental Research Institute of Michigan, Ann Arbor, Ml. Proceedings, 1: 407-418.

BOARDMAN JW, KRUSE FA \& GREEN RO. 1995. Mapping target signatures via partial unmixing of AVIRIS data. In: Annual JPL Airborne Geoscience Workshop, 5, Pasadena, CA. Summaries, JPL Publication 95(1): 23-26.

BROOKS RR, REEVES RD, BAKER AJM, RIZZO JA \& DIAZ FERREIRA H. 1990. The Brazilian Serpentine Plant Expedition (BRASPEX), 1988. National Geographic Research, 6: 205-219.

CARRERE V \& CONEL JE. 1993. Recovery of atmospheric water vapor total column abundance from imaging spectrometer data around $940 \mathrm{~nm}$ sensitivity analysis and application to Airborne Visible / Infrared Imaging
Spectrometer (AVIRIS) data. Remote Sensing of Environment, 44: 179204.

CARVALHO APF, CARVALHO JÚNIOR OA, GUIMARÃES RF, MADEIRA NETO JS \& BUSTAMANTE MMC. 2001. Measurement of color in hyperspectral images (AVIRIS) using the CIE (Commission Internationale D'éclairage) system. In: JPL Airborne Earth Science Workshop, 10, California, Pasadena. JPL Airborne Earth Science Workshop, 10, California, Pasadena. Proceedings, JPL Publication 02-1, p. 133-140.

CARVALHO APF, CARVALHO JÚNIOR OA, GUIMARÃES RF, MARTINS ES \& BUSTAMANTE MMC. 2002. Utilização do classificador Spectral Correlation Mapper em imagens TM-Landsat. Espaço e Geografia, 5(1): 219-232.

CARVALHO JÚNIOR OA, CARVALHO APF, GUIMARÃES RF, MENESES PR \& SHIMABUKURO Y. 2003. Mistura espectral: (I) detecção dos membros finais utilizando a geometria do Simplex. Espaço e Geografia, 6(1): 147-173

CARVALHO JÚNIOR OA, CARVALHO APF, MENESES PR \& GUIMARÃES RF. 2002. Classificação e eliminação dos ruídos em imagens hiperespectrais pela análise seqüencial da transformação por fração de ruído mínima. Revista Brasileira de Geofísica. 20(1): 31-41.

CARVALHO JÚNIOR OA, MARTINS ES, BAPTISTA GMM, CARVALHO APF, MADEIRA NETTO JS \& MENESES PR. 1999. Mineralogical differentiation in weathering profiles of lateritic Ni using AVIRIS data, in Niquelandia - G0, Brazil. In: JPL Airborne Earth Science Workshop, 8, Pasadena, CA. Summaries, JPL Publication 99-17: 3-11.

CHANDRASEKHAR S. 1960. Radioative transfer. Dover, Mineola, N.Y. $393 \mathrm{pp}$

CRAIG M. 1994. Minimum-volume transforms for remotely sensed data. IEEE Transactions on Geoscience and Remote Sensing, 32: 542-552.

ENVI. 2000. The environment for visualizing images users guide, 4 ed., Research Systems Inc., Boulder, C0, p. 930.

GAO BC, HEIDEBRECHT KB \& GOETZ AFH. 1993. Derivation of scaled surface reflectances from AVIRIS data. Remote Sensing of Environment, 44: $165-178$

GARCIA M \& USTIN SL. 2001. Detection of interannual vegetation responses to climatic variability using AVIRIS data in a coastal savanna in California. IEEE Transactions on Geoscience and Remote Sensing, 39(7): 1480-1490

GREEN AA, BERMAN M, SWITZER P \& CRAIG MD. 1988. A transformation for ordering multispectral data in terms of images quality with implications for noise removal: IEEE Transactions on Geoscience and Remote Sensing, 26(1): 65-74.

GREEN R0. 1990. Retrieval of reflectance from calibrated radiance imagery measured by the Airborne Visible/Infrared Imaging Spectrometer (AVIRIS) for lithological mapping of Clark Mountains, California. 
In: Annual JPL Airborne Visible/Infrared Imaging Spectrometer (AVIRIS) Workshop, 2, Pasadena, CA. Summaries, JPL Publication 90-54, p. 167-175.

GREEN R0. 1991. Retrieval of reflectance from AVIRIS - measured radiance using a radioative transfer code. In: Annual JPL Airborne Visible/Infrared Imaging Spectrometer (AVIRIS) Workshop, 3, Pasadena, CA, Summaries, JPL Publication 91-28, p. 200-210.

GREEN RO, CONEL JE, MARGOLIS JS, BRUEGGE J \& HOOVER GL. 1991. An inversion algorithm for retrieval at atmospheric and leaf water absorption from AVIRIS radiance with compensation for atmospheric scattering. In: Annual JPL Airborne Visible/Infrared Imaging Spectrometer (AVIRIS) Workshop, 3, Pasadena, CA, Summaries, JPL Publication 91-28, p. 51-61.

GREEN RO, CONEL JE \& ROBERTS DA. 1993. Estimation of aerosol optical depth, and additional atmospheric parameters for the calculation of apparent reflectance from radiance measured by the airborne visible/infrared imaging spectrometer. In: Annual JPL Airborne Earth Science Workshop, 4, Pasadena, CA, Summaries, JPL Publication 93-26, p. 83-86.

ISAACS RG \& VOGELMANN AM. 1988. Multispectral sensor data simulation based on the multiple scattering LOWTRAN Code. Remote Sensing of Environment, 26(10): 75-99.

KAUFMAN YJ, HOBBS PV, KIRCHHOFF VWJH, ARTAXO P, REMER LA, HOLBEN BN, KING MD, WARD DE, PRINS EM, LONGO KM, MATTOS LF, NOBRE CA, SPINHIRNE JD, JI 0, THOMPSON AM, GLEASON JF, CHRISTOPHER SA \& TSAY SC. 1998. Smoke, Clouds, and Radiation-Brazil (SCAR-B) experiment. J. Geophys. Res., 103(D24): 31.783-31.808.

KRUSEFA. 1996. Geologic mapping using combined optical remote sensing and SIR-C/X-SAR data. In: Thematic Conference and Workshops on Applied Geologic Remote Sensing, 11, Las Vegas, Nevada. Proceedings, 2: $142-150$.

KRUSE FA. 1999. Mapping hot spring deposits with AVIRIS at Steamboat Springs, Nevada. In: Annual JPL Airborne Earth Science Workshop, 8, Pasadena, CA. Summaries, JPL Publication 99-17, p. 239-245.

KRUSE FA \& BOARDMAN JW. 1999. Fifteen years of hyperspectral data: Northern Grapevine Mountains, Nevada. In: Annual JPL Airborne Earth Science Workshop, 5, Pasadena CA, Summaries, JPL Publication 99-17, p. 247-258.

KRUSE FA, HUNTINGTON JH \& GREEN RO. 1996. Results from the 1995 AVIRIS geology group shoot. In: Proceedings 2nd International Airborne Remote Sensing Conference and Exhibition, 1: 211-220.
LATORRE M, CARVALHO JÚNIOR OA, CARVALHO APF \& SHIMABUKURO YE. 2002. Correção atmostérica: conceitos e fundamentos Espaço \& Geografia. 5(1): 153-178.

RICHARDSON LL. 1996. Remote sensing of algal bloom dynamics. BioScience, 46(7): 492-501.

RICHARDSON LL, BUISON D, LUI CJ \& AMBROSIA V. 1994. The detection of algal photosynthetic accessory pigments using Airborne VisibleInfrared imaging Spectrometer (AVIRIS) Spectral Data: Marine. Tecnology Society Journal, 28: 10-21.

SCHMID B. 1996. Comparison of modeled and empirical approaches for retrieving columnar water vapour from solar transmittance measurements in the $0.94 \mathrm{~m}$ region. J. Geophys. Res., 101(D5): 27 april, p. 9.3459.358.

SMITH MO \& ADAMS JB. 1985a. Strategy for analyzing mixed pixels in remotely sensed imagery. In: NASA/JPL Aircraff SAR Workshop, Pasadena, CA. Summaries, JPL Publication 85-39, p. 47-48.

SMITH MO \& ADAMS JB. 1985b. Interpretation of AIS images of Cuprite, Nevada, using constrains of spectral mixtures. In: Aiborne Imaging Spectrometer Data Analysis Workshop, Pasadena, CA. Summaries, JPL Publication 85-41, p. 62-68.

SMITH MO, JOHNSON PE \& ADAMS JB. 1985. Quantitative determination of mineral types and abundances from reflectance spectra using principal components analysis. Proc. 15th Lunar Planet. Sci. Conf. Part2. J Geophys. Res., 90(Suppl): C797-C804.

TANRÉ D, DEROO C, DEHAUT P, HERMAN M, MORRCRETTE JJ, PERBOS J \& DESCHAMPS PY. 1986. Simulation of the Satellite Signal in the Solar Spectrum: User's Guide. L.0.A, Lille, 149 p.

TANRÉ D, DEROO C, DUHAUT P, HERMAN M, MORCRETTE JJ, PERBOS $J$ \& DESCHAMPS PY. 1990. Description of a Computer Code to Simulated the Satellite Signal in the solar Spectrum: the 5S Code. Int. J. Remote Sensing, 11(4): 659-668.

TOMPKINS S, MUSTARD JF, PIETERS CM \& FORSYTH DW. 1997. Optimization of endmembers mixture analysis for spectral. Remote Sensing of Environment, 59: 472-489.

VERMOTE E, TANRE D, DEUZE JL, HERMAN M \& MORCRETTE JJ. 1996. Second Simulation of the Satellite Signal in the Solar Spectrum (6S), 6S User's Guide Version 1. NASA-GSFC, Greenbelt, Maryland, 134 p.

WINTER M. 1999. Fast autonomous spectral endmember determination in hyperspectral data. In: Int. Conf. Applied Geologic Remote Sensing, 13, Vancouver, BC, Canada, 2: 337-344. 


\section{NOTAS SOBRE OS AUTORES}

Osmar Abílio de Carvalho Júnior. Recebeu o título de geólogo pela Universidade de Brasília em 1990, e de mestre e doutor em prospecção mineral pela Universidade de Brasília em 1995 e 2000, respectivamente. Trabalhou como Pesquisador do Instituto Nacional de Pesquisas Espaciais (INPE) entre 2002 e 2004 . Atualmente é professor da Universidade de Brasília e bolsista de produtividade e pesquisa do CNPq onde realiza pesquisas sobre o processamento digital de imagens multiespectrais e hiperespectrais.

Renato Fontes Guimarães. Recebeu o título de engenheiro cartógrafo pela Universidade Estadual do Rio de Janeiro em 1987, o grau de mestre em geofísica pelo Observatório Nacional em 1991 e o grau de doutor em geologia pela Universidade Federal do Rio de Janeiro em 2000. Atualmente é professor Adjunto do Departamento de Geografia e chefe do Laboratório de Sistemas de Informações Espaciais do Departamento de Geografia. Possui como principais linhas de pesquisa o sensoriamento remoto e a modelagem matemática dos processos erosivos. É bolsista de produtividade e pesquisa do CNPq

Éder de Souza Martins. Obteve a Graduação em Geologia pela Universidade de Brasília, UNB, em 1987. Realizou o mestrado em 1987 e doutorado 1999 ambos em Geologia pela Universidade de Brasília. Atualmente trabalha na Empresa Brasileira de Pesquisa Agropecuária, Centro de Pesquisa Agropecuária dos Cerrados, CPAC onde coordena o laboratório de pedologia. Realiza pesquisa em metodologias para o mapeamento e generalização cartográfica de informações pedológicas.

Ana Paula Ferreira de Carvalho. Recebeu o título de engenheira agronômica pela Universidade de Brasília em 1992, o grau de mestre em Ecologia pela Universidade de Brasília em 1998 e atualmente realiza o doutorado em bioquímica de plantas no Departamento de Ecologia da Universidade de Brasília. Trabalha na divisão de geoprocessamento do Instituto Nacional de Colonização e Reforma Agrária (INCRA).

Roberto Arnaldo Trancoso Gomes. Recebeu 0 título de geógrafo pela Universidade Federal do Rio de Janeiro em 1999, o grau de mestre em Geografia pela Universidade Federal do Rio de Janeiro em 2002 e atualmente realiza o doutorado utilizando modelagem matemática e sistema de informação geográfica no Departamento de Geografia da Universidade Federal do Rio de Janeiro. 